Enferm Bras 2019;18(2);303-13

https://doi.org/10.33233/eb.v18i2.2500

\title{
REVISÃo \\ Adesão de idosos hipertensos ao tratamento farmacológico
}

Isabel Lopes Pereira da Silva Amaral ${ }^{*}$, Ana Paula Silva de Brito Rodrigues**, Mariana Suênia Soares de Miranda*, Simônica Cruz Almeida de Carvalho*, Minéya Cabral da Silva*, Ana Carla Silva dos Santos, M.Sc. ${ }^{\star \star *}$

*Pós-Graduada em Saúde Pública com ênfase em Saúde da Família pela Faculdade de Comunicação e Turismo de Olinda (FACOTTUR), ${ }^{* * B a c h a r e l ~ e m ~ E n f e r m a g e m ~ p e l a ~ F a c u l d a d e ~}$ de Ciências Humanas de Olinda (FACHO), ${ }^{* * *}$ Mestre em Gerontologia: Atividade Física e Saúde, Docente do Instituto de Formação, Assessoria e Pesquisa (IFAP)

Recebido em 30 de agosto de 2018; aceito em 28 de abril de 2019.

Endereço de correspondência: Isabel Lopes Pereira da Silva Amaral, 1, Travessa Santa Catarina, 25, 53620-373 Igarassu PE, E-mail: isabel20ilps@yahoo.com.br; Ana Paula Silva de Brito Rodrigues: paulabrito784@gmail.com; Mariana Suênia Soares de Miranda: maryzinha_suenia@hotmail.com; Simônica Cruz Almeida de Carvalho: simonica_cruz@outlook.com; Minéya Cabral da Silva: mineyacs@gmail.com; Ana Carla Silva dos Santos: anycarll@hotmail.com

\section{Resumo}

Introdução: O aumento da população idosa leva ao consequente aumento da prevalência de doenças crônicas não transmissíveis, responsáveis pelas maiores causas de morbimortalidade mundial. Dentre as doenças crônicas a Hipertensão Arterial Sistêmica é responsável por aproximadamente 7,6 milhões de mortes ao ano no mundo. Objetivo: Sintetizar os principais resultados de pesquisas e analisar criticamente as evidências científicas relativas ao tema adesão do paciente idoso hipertenso ao tratamento farmacológico. Métodos: Revisão integrativa da literatura, realizada nas bases de dados: Medical Literature Analysis and Retrieval System Online (MEDLINE) e Literatura Latino-Americana e do Caribe em Ciências da Saúde (LILACS), Bases de Dados de Enfermagem (BDENF), por meio dos Descritores em Ciências da Saúde (DeCS): idosos, hipertensão e adesão à medicação, os quais foram integrados por meio do operador lógico booleano "AND", no período de 2010 a 2018, norteado pela seguinte questão: Quais fatores estão associados a não adesão dos idosos ao tratamento farmacológico antihipertensivo? Resultados: Dentre os artigos identificados, 12 estudos fizeram parte da amostra do estudo, as categorias que permitiram uma melhor apresentação das evidências científicas sobre adesão do paciente idoso hipertenso ao tratamento farmacológico foram: grau de instrução, situações estressantes, falta de vínculo com o profissional. Conclusão: Constatou-se que os fatores associados a não adesão dos idosos ao tratamento farmacológico foram: o grau de instrução, déficit cognitivo e síndrome da fragilidade; a falta de sintomas da doença, a politerapia, péssima caligrafia dos prescritores e a falta de vínculo com o profissional de saúde; bem como a presença de sintomas depressivos e eventos estressantes da vida.

Palavras-chave: idosos, hipertensão, adesão à medicação.

\begin{abstract}
Adherence of hypertensive elderly to pharmacological treatment

Introduction: The increase in the elderly population leads to a consequent increase in the prevalence of chronic noncommunicable diseases, responsible for the major causes of morbidity and mortality worldwide. Among chronic diseases, Systemic Arterial Hypertension accounts for approximately 7.6 million deaths per year worldwide. Objective: To synthesize the main research results and to critically analyze the scientific evidence related to the topic of adherence of elderly hypertensive patients to pharmacological treatment. Methods: Integrative literature review, carried out in the databases: Medical Literature Analysis and Retrieval System Online (MEDLINE) and Latin American and Caribbean Literature in Health Sciences (LILACS), Nursing Databases (BDENF), through of the Health Sciences Descriptors (DeCS): elderly, hypertension and medication adherence, which were integrated through the Boolean logical operator "AND", in the period 2010 to 2018, guided by the following question: What factors are associated with the non-
\end{abstract}


adherence of the elderly to antihypertensive pharmacological treatment? Results: Twelve studies were included, the categories that allowed a better presentation of the scientific evidences on adherence of the hypertensive elderly patient to the pharmacological treatment were: degree of education, stressful situations, lack of bond with the professional. Conclusion: We found that the factors associated with the non-adherence of the elderly to the pharmacological treatment were: the degree of education, cognitive deficit and fragility syndrome, the lack of symptoms of the disease, polytherapy, poor handwriting of prescribers and lack of bond with the health professional, as well as the presence of depressive symptoms and stressful life events.

Key-words: aged, hypertension, medication adherence.

\section{Resumen}

\section{Adhesión de mayores hipertensos al tratamiento farmacológico}

Introducción: El aumento de la población de adultos mayores lleva al consiguiente aumento de la prevalencia de enfermedades crónicas no transmisibles, responsables de las mayores causas de morbimortalidad mundial. Entre las enfermedades crónicas la Hipertensión Arterial Sistémica es responsable de aproximadamente 7,6 millones de muertes al año en el mundo. Objetivo: Sintetizar los principales resultados de investigaciones y analizar críticamente las evidencias científicas relativas al tema adhesión del paciente mayor hipertenso al tratamiento farmacológico. Métodos: Revisión integradora de la literatura realizada en las bases de datos: Medical Literature Analysis and Retrieval System Online (MEDLINE) y Literatura Latino-Americana e del Caribe en Ciencias de la Salud (LILACS), Bases de Dados de Enfermagem (BDENF), a través de los Descriptores en Ciencias de la Salud (DeCS): ancianos, hipertensión y adhesión a la medicación, los cuales fueron integrados por el operador lógico booleano "AND". Resultados: Se incluyeron 12 estudios, las categorías que permitieron una mejor presentación de las evidencias científicas sobre adhesión de los pacientes ancianos hipertensos al tratamiento farmacológico fueron: grado de instrucción, situaciones estresantes, falta de vínculo con el profesional. Conclusión: Se constató que los factores asociados a la no adhesión de los mayores al tratamiento farmacológico fueron: grado de instrucción, déficit cognitivo y síndrome de la fragilidad; la falta de síntomas de la enfermedad, la politerapia, mala caligrafía de los prescriptores y falta de vínculo con el profesional, así como presencia de síntomas depresivos y eventos estresantes de la vida. Palabras-clave: ancianos, hipertensión, adhesión a la medicación

Introdução

De acordo com dados do Instituto Brasileiro de Geografia e Estatística (IBGE), aproximadamente 20 milhões de pessoas no Brasil, possuem idade igual ou superior a 60 anos, caracterizando $10 \%$ da população do país como idosa. Para a Organização Mundial de Saúde (OMS), entre os anos de 1950 e 2025 a população idosa do Brasil deverá ter aumentado em quinze vezes, enquanto a população total em apenas cinco vezes. Nos países desenvolvidos esse processo aconteceu de forma lenta e associado à melhoria nas condições de vida, enquanto que no Brasil, um país em desenvolvimento, tem acontecido rapidamente, apresentando cerca de 32 milhões de pessoas com mais de 60 anos, sem melhoria na organização social e de saúde para atender à nova demanda [1].

O aumento da população idosa, leva ao consequente aumento da prevalência de doenças crônicas não transmissíveis (DCNT), responsáveis pelas maiores causas de morbimortalidade no mundo [2]. Dentre as doenças crônicas, pode-se destacar a Hipertensão Arterial Sistêmica (HAS), que é uma condição clínica multifatorial caracterizada por uma elevação sustentada dos níveis pressóricos maior ou igual a 140 e/ou $90 \mathrm{mmHg}$ [3].

A HAS é responsável por aproximadamente 7,6 milhões de mortes ao ano no mundo [4]. No Brasil, acomete $32,5 \%$ de indivíduos adultos e quando se trata de idosos a HAS atinge mais de $60 \%$ [3]. Além de a doença ser considerada fator de risco, pois está frequentemente associada às alterações funcionais e/ou estruturais de órgãos alvos como coração, encéfalo, rins e vasos sanguíneos, bem como associada às alterações metabólicas e ao aumento progressivo do risco de eventos cardiovasculares [5].

Dados norte-americanos de 2015 revelaram que a HAS estava presente em 69\% pacientes com primeiro episódio de Infarto Agudo do Miocárdio (IAM), 77\% de Acidente Vascular Encefálico (AVE), 75\% com Insuficiência Cardíaca (IC) e 60\% com Doença Arterial Periférica (DAP). A HAS é responsável por $45 \%$ das mortes cardíacas e $51 \%$ das mortes decorrentes de AVE [3]. 
Por se tratar de um importante fator de morbimortalidade, principalmente em idosos, a HAS necessita de tratamento adequado, seja adotando medidas não farmacológicas como mudanças na dieta, prática de atividades físicas, o não consumo de tabaco e álcool entre outros, como também exige adoção e manutenção rigorosa do tratamento farmacológico. A finalidade do tratamento farmacológico inclui a redução ou controle dos níveis pressóricos, diminuição do risco de doenças do aparelho circulatório bem como melhor qualidade de vida [4].

A adesão ao tratamento medicamentoso está relacionada ao grau de cumprimento da terapia indicada exatamente da forma que foi proposta pelo profissional de saúde. É um processo complexo e comportamental, e pode estar associado a diversos fatores, pois o paciente idoso precisa conscientizar-se de sua condição clínica e comprometer-se com o tratamento, o apoio familiar e a clareza nas recomendações dos profissionais de saúde são também imprescindíveis para sucesso do tratamento [6].

Estudos realizados observaram que alguns pacientes com HAS não alcançaram o controle dos níveis pressóricos mesmo recebendo tratamento e sendo acompanhados por serviços de saúde, fato que pressupõe a não adesão ao tratamento ou monitoramento inadequado [7]. A adesão à medicação por vezes é imprescindível para os indivíduos hipertensos, mas na prática clínica observa-se o abandono à terapêutica medicamentosa por pelo menos 16 a $50 \%$ dos pacientes no primeiro ano de tratamento, o que leva ao descontrole da doença e complicações bem como retardo de agravos [6].

Considerando a relevância da temática da adesão dos idosos hipertensos ao tratamento farmacológico, é inegável a contribuição de estudos que busquem disseminar as publicações, em particular, na área de saúde, fornecendo subsídios científicos aos profissionais de saúde para orientar as ações de prevenção e consequentemente diminuição dos gastos públicos.

Diante do exposto surge a seguinte questão norteadora do estudo: Quais fatores estão associados a não adesão dos idosos ao tratamento farmacológico anti-hipertensivo? Para responder à questão norteadora, o estudo tem como objetivo analisar a produção científica sobre a referida temática, disponibilizada em periódicos online.

Material e métodos

Esta pesquisa é do tipo revisão integrativa da literatura, desenvolvida através de artigos oriundos das bases de dados eletrônicas de artigos indexados. As etapas para a realização do estudo foram: 1) identificação do tema e questão condutora do estudo, 2) escolha dos descritores; 3) critérios de inclusão e exclusão dos artigos; 4) definição das informações a serem retiradas dos estudos selecionados; 5) avaliação dos artigos incluídos na revisão; 6) interpretação dos resultados e discussão.

Para nortear a revisão integrativa da literatura, foi formulada a seguinte questão: Quais fatores estão associados a não adesão dos idosos ao tratamento farmacológico anti-hipertensivo?

A coleta de dados realizou-se no mês de abril de 2018, com limite de período entre os anos de 2010 a 2018, disponíveis na Biblioteca Virtual em Saúde (BVS), através das seguintes bases de dados: Bases de Dados de Enfermagem (BDENF), Medical Literature Analysis and Retrieval System Online (MEDLINE) e Literatura Latino-Americana e do Caribe em Ciências da Saúde (LILACS), por meio dos Descritores em Ciências da Saúde (DeCS): idosos, hipertensão e adesão à medicação, os quais foram integrados por meio do operador lógico booleano "AND".

Em virtude das características específicas para o acesso às bases de dados selecionadas, as estratégias utilizadas para localizar os artigos foram adaptadas para cada uma, tendo como eixo norteador a pergunta e os critérios de inclusão da revisão integrativa, previamente estabelecidos para manter a coerência na busca dos artigos e evitar possíveis vieses.

Os artigos foram selecionados pelos seguintes critérios de inclusão: 1) artigos disponíveis nas bases de dados com acesso livre e gratuito, 2) textos completos, 3) língua portuguesa, inglesa e espanhola, 4) com o período de publicação entre os anos de 2008 a 2017, 5) amostra constituída por idoso masculino e feminino, 6) o tipo de documento teria que ser artigo científico que respondesse à questão do estudo. Os critérios de exclusão foram: 1) artigos que não estivessem disponíveis na íntegra de forma gratuita, 2) teses, 3) documentos de projeto, 4) trabalhos apresentados em congresso e conferência, 5) monografias e artigos que não respondam a pergunta norteadora a partir da leitura do resumo, 6) artigos em que os dados fossem coletados em prontuários ou banco de dados de farmácias, 7) artigos em que obrigatoriamente o idoso tivesse que ter outras doenças crônicas além da hipertensão. 
A apresentação dos resultados foi realizada de forma descritiva, por meio de figuras e tabelas possibilitando ao leitor a avaliação da aplicabilidade da revisão integrativa.

Por não se enquadrar em uma pesquisa que envolve seres humanos, o presente estudo não necessita de apreciação do Comitê de Ética em Pesquisa, nem tampouco utilização de Termo de Consentimento Livre e Esclarecido (TCLE).

\section{Resultados e discussão}

Na presente revisão, após seleção dos estudos analisaram-se 12 artigos (Figura 1). A caracterização dos estudos que compuseram a revisão pode ser observada na Tabela I.

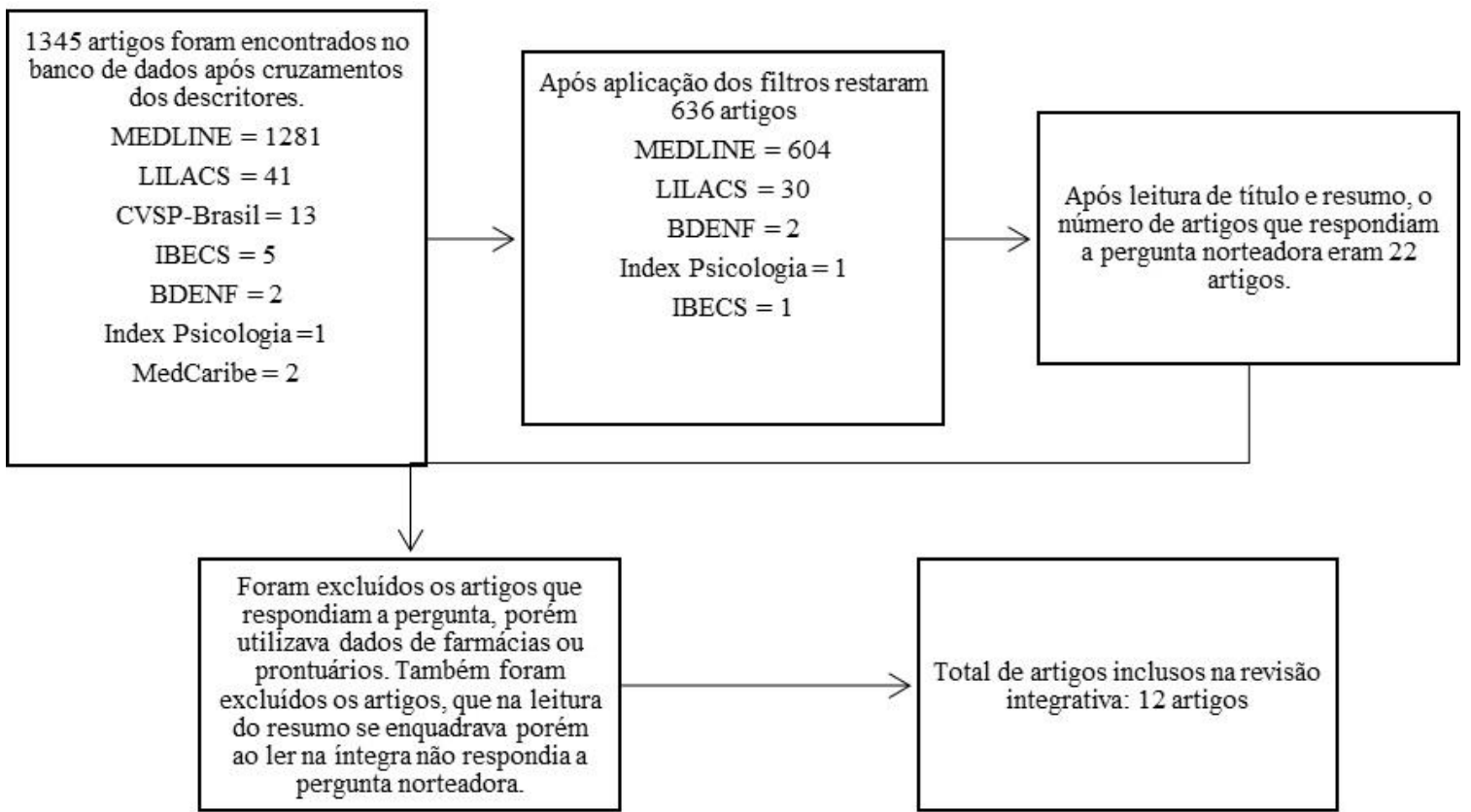

Figura 1 - Fluxograma dos estudos contemplados na pesquisa segundo os critérios de elegibilidade.

Tabela I - Caracterização dos estudos que compuseram a revisão integrativa. (ver PDF em anexo).

Após a leitura dos artigos selecionados para revisão integrativa, foi possível perceber que existem diversos fatores relacionados com a adesão ou não adesão de idosos hipertensos ao tratamento farmacológico. Em relação ao sexo, 100\% dos artigos incluídos na presente revisão tinham como maior parte de sua amostra o sexo feminino. O artigo "Tô sentindo nada": Percepções de pacientes idosos sobre o tratamento da hipertensão arterial sistêmica" separou a amostra em dois grupos, onde G1 era o grupo de idosos aderentes ao tratamento e G2 o grupo de idosos não aderentes ao tratamento. Em ambos os grupos o percentual de mulheres foi maior [13]. Mulheres geralmente se preocupam mais com a saúde, e são elas que procuram os serviços de Hiperdia e solicitam medicamentos para si e seus companheiros [17].

Os artigos "Adesão ao uso de medicamentos entre idosos hipertensos" e "Qualidade de vida e adesão ao tratamento farmacológico entre idosos hipertensos" também tinham dois grupos, um referente aos aderentes à medicação e outro para os não aderentes. Nesses artigos também foi possível evidenciar que o número de mulheres nos grupos foi superior ao número de homens. Pode-se concluir que o sexo não é um fator relacionado à baixa adesão, porém caracteriza a feminização do envelhecimento [10,11].

Em relação à etnia não houve uma diferença exorbitante, os artigos trazem dados diversificados dessa variável. Em alguns, prevaleceram pacientes cujo tom de pele é branco, seguido de pardos e negros. O artigo "Adesão ao tratamento e controle da pressão arterial em idosos com hipertensão" relata que $60 \%$ da amostra se autodeclarava branco, e no artigo "Fatores associados à adesão ao tratamento farmacológico em idosos que utilizam medicamento 
anti-hipertensivo" teve 45,5\% autodeclarados brancos [9,15]. Dos 25 usuários da Unidade Básica de Saúde entrevistados no artigo "Adesão ao tratamento de idosos com hipertensão em uma unidade básica de saúde de João Pessoa, Estado da Paraíba", apenas 3 eram negros. Contrariando a literatura que diz que hipoteticamente há maior predisposição genética em negros para desenvolverem a hipertensão arterial sistêmica [17].

Pode-se perceber que a maioria dos estudos apontou a média ou variação de idades acima de 70 anos. O artigo "Adesão ao uso de medicamentos entre idosos hipertensos" teve como média de idade 71,7 anos dos idosos entrevistados. Neste estudo, a taxa de adesão foi de $31,45 \%$. Os idosos mais jovens apresentam maior independência e autonomia, por não serem alvo de prestação de cuidados de terceiros, com isso favorece o abandono ao tratamento, ou seja, deixar de seguir a prescrição médica como determinada pelo profissional de saúde conhecida como "não adesão intencional" [11].

Dos estudos selecionados para esta revisão integrativa, concernente ao estado civil quando era citado, pode-se perceber que mais da metade da amostra dos artigos eram casados ou residiam com alguém. O artigo "Qualidade de vida e adesão ao tratamento farmacológico entre idosos hipertensos" coletou dados a partir de entrevista com 1.029 idosos, dos quais 50,9\% eram aderentes ao tratamento e $49,1 \%$ não aderentes. Em ambos os grupos deste estudo houve predominância de pessoas casadas ou que residiam com companheiros. Desta forma, o estado civil não apresentou associação significativa com a baixa adesão ao tratamento farmacológico [10].

Sobre o grau de instrução, em alguns artigos não foi possível verificar relação à adesão ou não adesão, porém o artigo "Adesão ao tratamento de idosos com hipertensão em uma unidade básica de saúde de João Pessoa, Estado da Paraíba" teve maior parte de sua amostra com grau de escolaridade até $3^{\text {a }}$ série. Dos 25 participantes da pesquisa, 13 não utilizavam corretamente a medicação para controle da pressão arterial. $O$ nível de escolaridade está diretamente associado à temática. A doença é mais prevalente em indivíduos com baixa escolaridade, pois muitas vezes abandonam o tratamento por falta de senso crítico e de entendimento sobre a própria patologia que o acomete [17].

O déficit cognitivo foi relatado em alguns estudos e consideravelmente associado a baixos escores de adesão à medicação, no artigo "Adesão ao uso de medicamento em idosos hipertensos", um grupo de 124 idosos foram entrevistados. Deste grupo, 85 idosos foram classificados como não aderentes, caracterizando 68,5\% da amostra. Dos idosos não aderentes, $61,8 \%(n=47)$ apresentaram déficit cognitivo após aplicação do Miniexame do Estado Mental em associação com a Escala de Autoadesão [11].

A síndrome da fragilidade é um problema importante em pessoas idosas e constantemente são criados instrumentos especializados em diagnósticos e desenvolvimento de medidas preventivas, já que a fragilidade exerce consequências negativas não apenas no resultado da doença, mas também na adesão ao tratamento [8].

O artigo "Efeito da síndrome da fragilidade na adesão ao tratamento em idosos hipertensos", com o intuito de analisar o efeito dessa síndrome no tratamento de idosos hipertensos, entrevistou 300 idosos hospitalizados devido às urgências hipertensivas. A síndrome da fragilidade foi identificada em 197 idosos, percentual de $65.67 \%$ da amostra. Neste estudo $50 \%$ dos idosos não aderiram ao tratamento, com cumprimento adequado da prescrição médica [8]. No artigo "Fatores associados à adesão ao tratamento farmacológico em idosos que utilizam medicamento anti-hipertensivo" a não classificação como idoso frágil, permaneceu estatisticamente associada à adesão à terapia farmacológica [9].

A politerapia é um fator precipitante para a não adesão ou abandono ao tratamento farmacológico, indivíduos com múltiplas comorbidades são menos propensos a cumprir seus planos de tratamento devido ao maior número de medicamentos [13]. O artigo "Qualidade de vida e adesão ao tratamento farmacológico em idosos hipertensos" evidenciou que os idosos com maior número de comorbidades são menos propensos à adesão, devido a um maior número de fármacos e conhecimento inadequado sobre terapêutica. Quanto maior o número de medicamentos prescritos, maior o risco de potenciais interações perigosas e efeitos adversos, resultando em baixa adesão ao tratamento [15].

A não adesão pode estar relacionada ao fato de não "sentir nada", ou até mesmo a percepção de efeitos adversos. O artigo "Adesão ao tratamento e controle da pressão arterial em idosos com hipertensão" revela que $78 \%$ de sua amostra relatou não entender a letra do médico e isso era um obstáculo para cumprir a prescrição médica, $58 \%$ relataram interrupção da medicação devido a efeitos adversos [11]. O artigo "“Tô sentindo nada”: percepções de pacientes idosos sobre o tratamento da hipertensão arterial sistêmica" demonstrou que os pacientes que 
participaram da pesquisa relataram esquecimento das medicações por não estarem apresentando sintomas de pressão arterial elevada. Isso leva o paciente a desistir da terapia, já que "sentir nada" está associado a ausência de doença [13].

O artigo "Diferenças entre os sexos nas barreiras à adesão à medicação anti-hipertensiva: achados do estudo de coorte sobre adesão à medicação entre idosos (CoSMO)" avaliou alguns fatores que poderiam estar associados a uma maior ou menor adesão em homens e mulheres. A prevalência de baixos escores de adesão à medicação não diferiu de acordo com o sexo. Em modelos multivariáveis específicos do sexo, ter problemas com o custo da medicação e praticar menos modificações no estilo de vida para o controle da pressão arterial foram associados a baixos escores de adesão entre homens e mulheres. Fatores associados a baixos escores de adesão em homens, mas não em mulheres, incluíram redução da função sexual e IMC $\geq 25$. Fatores associados aos baixos escores de adesão nas mulheres, mas não nos homens, incluíram a insatisfação com a comunicação com o profissional de saúde [12]. O artigo "Adesão ao tratamento de idosos com hipertensão em uma unidade básica de saúde de João Pessoa, Estado da Paraíba" diz que para o sucesso da terapia, além dos esforços individuais é necessário que haja vínculo do profissional de saúde e o paciente. O enfermeiro exerce papel importante na adesão ao tratamento, pois em sua consulta estabelece um elo com o paciente facilitando a implementação de ações necessárias para adesão [17].

Fatores psicossociais desempenham um papel importante no comportamento de tomada de medicação dos pacientes. $\mathrm{O}$ artigo "Acontecimentos da vida, coping e adesão à medicação anti-hipertensiva entre adultos mais velhos" acrescenta evidências de que a ocorrência de eventos de vida pode impactar a adesão à medicação em pacientes idosos com hipertensão. Entre os participantes com níveis mais baixos de enfrentamento, aqueles que relataram uma mudança no estado financeiro, uma mudança na saúde ou comportamento de um membro da família e mudanças nas atividades recreativas, atividades da igreja, atividades sociais, atividades para dormir ou hábitos alimentares tiveram adesão significativamente menor do que aqueles que não tinham experimentado cada um desses eventos. A tristeza esmagadora decorrente de alguns tipos de eventos de vida pode então afetar a adesão e ao mesmo tempo desencadear um episódio de depressão [14].

Os sintomas depressivos são abordados no artigo "Associação de depressão com aderência de medicação anti-hipertensiva em adultos idosos: achados transversais e longitudinais do CoSMO". No início do estudo, 14,1\% dos participantes relataram baixa adesão aos medicamentos anti-hipertensivos, $13,0 \%$ relataram sintomas depressivos e 33,9\% relataram baixo apoio social. Um percentual maior de pessoas com sintomas depressivos versus sem sintomas ocorreu com mulheres, negras, não casadas, com menos de um ano de ensino médio, duas ou mais comorbidades e tendo um número maior de classes de medicamentos antihipertensivos [18].

Conclusão

Nas bases de dados pesquisadas foram encontrados e selecionados artigos, segundo a pergunta de pesquisa elaborada. Dentre os estudos selecionados constatou-se que os fatores associados a não adesão dos idosos ao tratamento farmacológico foram: o grau de instrução, déficit cognitivo e síndrome da fragilidade; a falta de sintomas da doença, a politerapia, péssima caligrafia dos prescritores e a falta de vínculo com o profissional de saúde; bem como a presença de sintomas depressivos e eventos estressantes da vida.

Ressalta-se que a presença de um cuidador ou membro da família junto ao idoso facilita a adesão, bem como um vínculo agradável com o profissional de saúde que prescreve os medicamentos. Pelo fato de serem idosos, mostram-se desmotivados devido a pouca perspectiva de vida.

Espera-se que este estudo possa auxiliar em pesquisas futuras que envolvam idosos, além de promover subsídios para criação de ações de prevenção da hipertensão e ações que busquem estimular os idosos a adesão terapêutica. 
1. Brasil. Atenção à Saúde da Pessoa Idosa e Envelhecimento; Série: Pactos pela saúde; 1 ed. Brasília: Ministério da Saúde; 2010.

2. Freitas JGA, Nielson SEO, Porto CC. Adesão ao tratamento farmacológico em idosos hipertensos: Uma revisão integrativa da literatura. Rev Soc Bras Clin Med 2015;13(1);75-84.

3. Brasil. Sociedade Brasileira de Cardiologia. $7^{\underline{a}}$ Diretriz Brasileira de Hipertensão Arterial. Arq Bras Cardiol 2016;107(3):1-83.

4. Brasil. Ministério da Saúde. Cadernos de Atenção Básica. Estratégias para o cuidado da pessoa com doenças crônica; Hipertensão Arterial Sistêmica, n 37; 2013.

5. Souza AO, Yamaguchi MU. Adesão e não adesão dos idosos ao tratamento antihipertensivo. Saúde e Pesquisa 2015;8;113-22.

6. Daniel ACQG, Veiga EV. Fatores que interferem na adesão terapêutica medicamentosa em hipertensos. Einstein 2013;11(3):331-7.

7. Silva CS, Paes NA, Figueiredo TMRM, Cardoso MAA, Silva ATMC, Araújo J SS. Controle pressórico e adesão/vínculo em hipertensos usuários da atenção primária à saúde. Physis Rev Esc Enferm USP 2013;47(03):584-90. https://doi.org/10.1590/s0080-623420130000300009

8. Chudiak A, Jankowska-Polańska B, Uchmanowicz I. Effect of frailty syndrome on treatment compliance in older hypertensive patients. Clin Interv Aging 2017;2017(12):805-14.

9. Aquino GA, Cruz DT, Silvério MS, Vieira MT, Bastos RR, Leite ICG. Fatores associados à adesão ao tratamento farmacológico em idosos que utilizam medicamento antihipertensivo. Rev Bras Geriatr Gerontol 2017;20(01):116-27. https://doi.org/10.1590/1981-22562017020.160098

10. Tavares DMS, Guimarães MO, Ferreira PCS, Dias FA, Martins NPF, Rodrigues LR. Qualidade de vida e adesão ao tratamento farmacológico entre idosos hipertensos. Rev Bras Enferm 2016;69(01):134-41. https://doi.org/10.1590/0034-7167.2016690118i

11. Aiolfi CR, Alvarenga MRM, Moura CS, Renovato RD. Adesão ao uso de medicamentos entre idosos hipertensos. Rev Bras Geriatr Gerontol 2015;18 (2):397-404. https://doi.org/10.1590/1809-9823.2015.14035

12. Holt EW, Muntner P, Joyce CJ, Donald EM, Larry SW, Krousel-Wood M. Sex differences in barriers to antihypertensive medication adherence: findings from the cohort study of medication adherence among older adults (CoSMO). J Am Geriatr Soc 2013;61(4):508-64. https://doi.org/10.1111/jgs.12171

13. Silva LOL, Soares MM, Oliveira MA, Rodrigues SM, Machado CJ, Dias CA. "Tô sentindo nada": percepções de pacientes idosos sobre tratamento da hipertensão arterial sistêmica. Rev Saúde Colet 2013;23(01):227-42. https://doi.org/10.1590/S010373312013000100013

14. Holt EW, Muntner P, Joyce C, Moriky DE, Webber LS, Wood MK. Events, coping, and antihypertensive medication adherence among older adults. Am J Epidemiol 2012;176(7):64-71. https://doi.org/10.1093/aje/kws233

15. Barbosa RGB, Ferrioli E, Morigute JC, Nogueira CB, Nobre F, Ueta J, Lima NKC. Adesão ao tratamento e controle da pressão arterial em idosos com hipertensão. Arq Bras Cardiol 2012;99(01):636-41. https://doi.org/10.1590/S0066-782X2012005000054

16. Wood MK, Joyce C, Holt E, Muntner P, Webber LS, Morisky DE, et al. Predictors of decline in medication adherence: Results from CoSMO. Hypertension 2012;58(5):80410. https://doi.org/10.1161/HYPERTENSIONAHA.111.176859

17. Dourado CS, Macêdo-Costa KNF, Oliveira JS, Leadebal ODCP, Silva GRF. Adesão ao tratamento de idosos com hipertensão em uma unidade básica de saúde de João Pessoa, Estado da Paraíba. Acta Scientiarum Health Sciences 2011;33(1):9-17. https://doi.org/10.4025/actascihealthsci.v33i1.7708

18. Krousel-Wood M, Islam T, Muntner P, Holt E, Joyse C, Morisky DE. Association of depression with antihypertensive medication adherence in older adults: cross-sectional and longitudinal findings from CoSMO. Ann Behav Med 2010;58(1):248-57. https://doi.org/10.1007/s12160-010-9217-1

19. Krousel-Wood M, Islam T, Morisky DE, Webber LS. Barriers to and determinants of medication adherence in hypertension management: perspective of the cohort study of medication adherence among older adults (CoSMO). Med Clin North Am 2009;93(3):753-69. 